\title{
ANALYSIS OF HALF VALUE LAYER (HVL), TENTH VALUE LAYER (TVL) AND MEAN FREE PATH (MFP) OF SOME OXIDES IN THE ENERGY RANGE OF 122KeV to $1330 \mathrm{KeV}$
}

\author{
PRADIP S. DAHINDE ${ }^{\mathrm{a} 1}$, G.P.DAPKE ${ }^{\mathrm{b}}$, S.D.RAUT ${ }^{\mathrm{c}}$, R.R.BHOSALE ${ }^{\mathrm{d}}$ AND PRAVINA P. PAWAR $^{\mathrm{e}}$

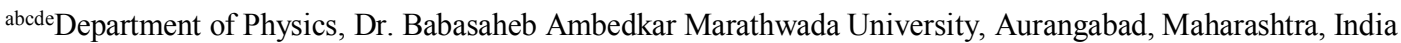

\begin{abstract}
In the present work half value layer (HVL), tenth value layer (TVL)and mean free path (Mfp) are the most frequently used important parameter for explaining both the penetrating ability of particular radiations and the penetration through specific objects or material. The half value layers (HVL), tenth value layers (TVL) gives the thickness of a shield or an absorber that reduces the radiation level by a factor of one-half and one tenth of the initial level, respectively. The concepts of HVL and TVL are widely used in shielding design. In the present investigation we calculated the values of linear attenuation coefficient, mean free path (MfP), half value layer (HVL) and tenth value layer (TVL) for some oxides such as aluminum oxide, manganese oxide, copper oxide and magnesium oxide in the energy range from $122 \mathrm{keV}$ to $1330 \mathrm{keV}$ were measured. The transmitted intensity of photons emitted by radioactive isotopes of $133 \mathrm{Ba}, 22 \mathrm{Na}, 137 \mathrm{Cs}$, $54 \mathrm{Mn}$ and $60 \mathrm{Co}$ measured using the $\mathrm{NaI}(\mathrm{TI})$ scintillation detector and results obtained are in good agreement with the values reported in the present study.
\end{abstract}

KEYWORDS: Linear Attenuation Coefficient; Mean Free Path; Half Value Layer; Tenth Value Layer

In recent years the study of interactions of radiation with different material has become an important in our routine life, especially in medical applications and it gives the increasing use of radioactive isotopes in different fields. Data on the attenuation of gamma-rays with matter is extremely useful for many scientific, agriculture, engineering, shielding and medical applications (Singh et al., 2006). The linear attenuation coefficient is extremely useful for characterizing the penetration and diffusion of gamma rays in the medium through which they passes. This mainly based on the photon energy, the nature of the selected material and the density of medium (Bashter, 1997). The interaction of photon of gamma radiation takes place various kinds of processes in the medium like photoelectric effect, incoherent scattering, coherent scattering and pair production and which is based on the energy of photon (Berger and Hubbell, 1987). The linear attenuation coefficient values of partial photon interaction processes such as photoelectric effect, Compton scattering, pair production and all these are available in the form of software package XCOM from Berger and Hubbell (1987). It is observed that the value of the linear attenuation coefficient depends on the incident photon energy and also depends on density $\rho$.

Now days the study of photon interaction with different materials has becomes more importance because of extensive use of radioactive sources in different field like medicine, industrial, biological, chemical and other field. The Oxide material covers a wide range of applications almost in every field. The study on the interaction of gamma radiation with selected oxide samples are of great interest from theoretical and experimental point of view. The linear attenuation coefficient of aluminum, manganese, copper, magnesium oxides in the energy range of 122-1330 $\mathrm{keV}$ are calculated. These values are calculated and compared with theoretical values calculated using XCOM program (Berger and Hubbell 1987 \& 1999) are found to be good agreement between each other. Absorbed radiation can cause biological damage in living tissues and DNA Shielding is the basic very useful method for radiation protection (Akkaş, 2015).

It is not possible to attenuate the radiation completely, the radiation shielding properties should also be known. Half-value layer (HVL), tenth-value layer (TVL), and mean free path $(\mathrm{mfp})$ are very essential theoretical parameters that are measured for gamma-ray shielding (Gundogdu et al., 2016). The HVL represents the thickness of an absorber that will reduce the gamma radiation to half, while the TVL represents the thickness of an absorber that will reduce the gamma radiation to a tenth of its original intensity (Akkurt et al., 2010). In this present investigation the HVL, TVL and mfp values for oxides are calculated for the energy of $122 \mathrm{KeV}$ to $1330 \mathrm{KeV}$. HVL and TVL values are very useful parameter for understanding the penetrating ability of particular radiations through specific material. 
DAHINDE AND PAWAR: ANALYSIS OF HALF VALUE LAYER (HVL), TENTH VALUE LAYER (TVL) AND MEAN...

\section{CALCULATION METHODS}

\section{Mass Attenuation Coefficient}

The inverse exponential power law that in the present work we study some theoretical parameters of some oxide that have been used to determine the mass attenuation coefficient $\mu_{m}$ and other related parameters which are based on it. A parallel beam of the measured intensity $I$ of the transmitted mono-energetic X-ray or $\gamma$-photons passing through matter is related to the incident intensity $I_{0}$ is usually referred to as Beer-Lambert law is given by the relation.

$$
I=I_{0} e^{-\mu_{m} X}
$$

Where, $I_{0}$ and $I$ are incident and transmitted photon intensities respectively,

$X$ is mass per unit area $\left(\mathrm{g} / \mathrm{cm}^{2}\right), \mu_{m}$ is mass attenuation coefficient $\left(\mathrm{cm}^{2} / \mathrm{g}\right)$ given by the following equation for a compound or mixture of elements (Jackson and Hawkes, 1981) (Hubbell and Seltzer, 1995): Solving the Eq. (1), we get the following equation for the linear attenuation coefficient $\left(\mathrm{cm}^{-1}\right)$ :

$$
\mu=1 / \operatorname{tln}(I / I o)
$$

\section{Half Value Layer (HVL) and Tenth Value Layer (TVL)}

The values of HVL and TVL thicknesses are calculated by using Eq.4 and Eq.5, respectively. The HVL and TVL are very important factor for calculations of sheling.

$$
\begin{aligned}
& H V L=X_{h}=\ln 2 / \mu, \\
& T V L=X_{t}=\ln 10 / \mu .
\end{aligned}
$$

\section{Mean Free Path}

Mean free path Mean free path is the average distance at which a single particle travels through the medium of given sample before interacting it with material and is calculated by the following equation

$$
X_{m}=1 / \mu \text {. }
$$

\section{EXPERIMENTAL DETAILS}

We measured incident and transmitted photon energies by using a narrow-beam good geometry set up. Figure 1 shows the schematic view of experimental set up. In this study we use the radioactive sources, $\mathrm{Co}^{57}$ (122
$\mathrm{keV}), \mathrm{Ba}^{133}$ (356 keV), $\mathrm{Na}^{22}$ (511 and $\left.1275 \mathrm{keV}\right), \mathrm{Cs}^{137}$ (662 $\mathrm{keV}), \mathrm{Mn}^{54}(840 \mathrm{keV})$ and $\mathrm{Co}^{60}(1170$ and $1330 \mathrm{keV})$ which is obtained from Bhabha Atomic Research Centre, Mumbai, India. The emission of gamma radiation by these radioactive sources were collimated and detected with the help of $\mathrm{NaI}(\mathrm{Tl})$ scintillation detector. The Signals from the detector $\left(2^{\prime \prime} \times 2 "\right) \mathrm{NaI}(\mathrm{Tl})$ crystal having energy resolution of $8.2 \%$ at $0.662 \mathrm{MeV}$ gamma rays from the decay of $\mathrm{Cs}^{137}$ after suitable amplification were recorded in an EG\&G ORTEC 13-bit plug-in-card coupled with a PC/AT. Stability and reproducibility of the arrangement were checked before and after each set of runs. In order to minimize the effects of small-angle scattering and multiple scattering events on the measured intensity, the transmitted intensity was measured by setting the channels at the fullwidth half-maximum position of the photo-peak.

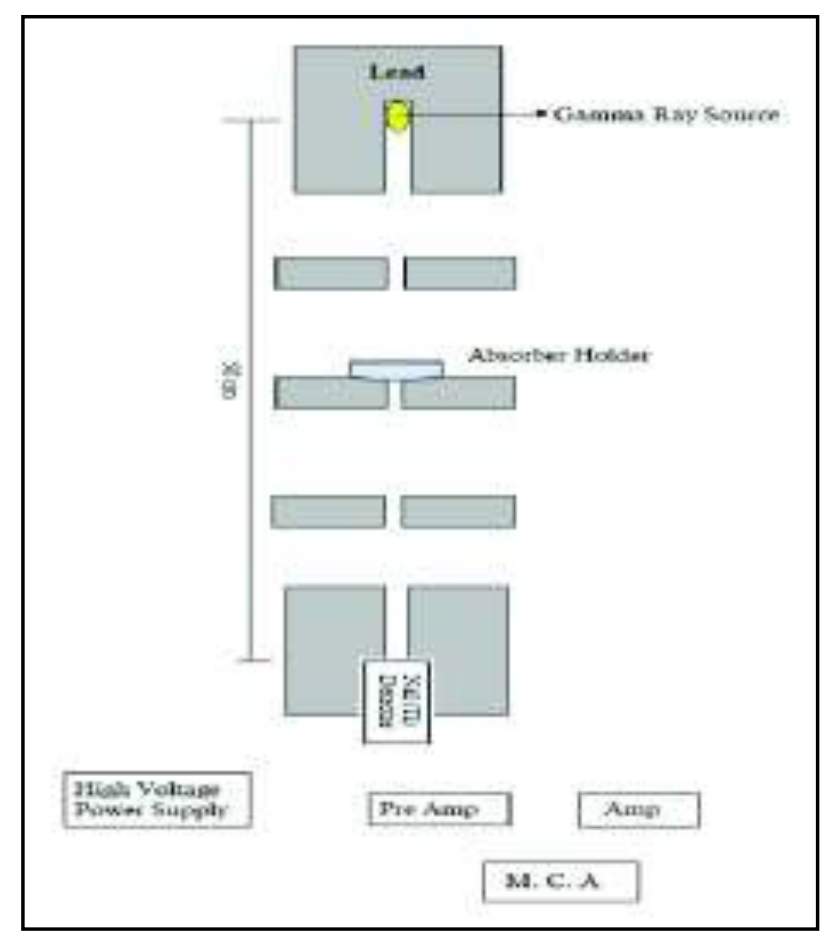

Figure 1: Narrow-beam Good Geometry Set-up

Pellet shaped uniform thickness of chosen oxides such as aluminum oxide $\left(\mathrm{Al}_{2} \mathrm{O}_{3}\right)$, manganese oxide $\left(\mathrm{MnO}_{2}\right)$, copper oxide $(\mathrm{CuO})$, magnesium oxide $(\mathrm{MgO})$ under investigation were confined in a cylindrical plastic container with diameter similar to that of the sample pellet. The diameters of the sample pellets were determined using a traveling microscope. The attenuation of photons in the empty containers was negligible. Each sample pellet was 
weighted in a sensitive digital balance with an accuracy of $0.001 \mathrm{mg}$ several times to obtain the average value of the mass. The mass per unit area was determined in each case using the diameter of the pellet and mean value of the mass of the pellet. The sample thickness was selected in order to satisfy the following ideal condition as far as possible (Creagh, 1987):

$$
2 \leq \ln \left(\frac{I_{o}}{I}\right) \leq 4
$$

The Mass attenuation coefficients $(\mu / \rho)$ of all the samples of oxides were determined from the measured values of incident photon intensity $I_{0}$ (without sample) and transmitted photon intensity $I$ that is with samples and mean values are used for the calculation of linear attenuation coefficients $(\mu)$ for all selected oxide sample. The experiments were conducted in an air-conditioned room to avoid possible shifts of the photo-peaks. Room temperature of $26 \pm 1{ }^{\circ} \mathrm{C}$ was maintained throughout the experimental period. Other sources of error, excluding multiple scattering and counting statistics, are small-angle scattering, sample impurity, nonuniformity of the sample, photo built-up effects, dead time of the counting instrument, and pulse pile effect which were evaluated and reduced.

The scattering angle was maintained in this study $<30 \mathrm{~min}$ by proper adjustment the distance between the detector and source $(30 \mathrm{~cm}<\mathrm{d}<50 \mathrm{~cm})$, due to the coherent and incoherent scattering at an angles calculated in the cross sections at intermediate energy range is very less or negligibly small (Hubbell, 1999). Hence, no small-angle scattering corrections were applied to the measured data. All the four oxides samples used in this study were of high quality sigma Aldrich and of high purity (99.9\%) without high-Z impurities. Hence, sample impurity corrections were not applied to the measured data.

In the presented investigation, uncertainty in the mass per unit area and the error due to nonuniformity of the sample are $<0.05 \%$ for all energies of interest. Optimum values of count rate and counting time were chosen to reduce the effects of photon built-up and pulse piles. The photon built-up effect, which is a consequence of the multiple scattering inside the sample, depends on the atomic number and sample thickness, as well as the incident photon energy. A built-in provision for dead time correction was present in the MCA used during this investigation.

\section{RESULTS AND DISCUSSION}

In the present work, the values of linear attenuation coefficient $\mu$ of investigated oxides such as aluminum oxide, manganese oxide, copper oxide, magnesium oxide. The variation between experimental and theoretical values of $\mu\left(\mathrm{cm}^{-1}\right)$ for all sample studied at 122 , $360,511,662,840,1170,1275-$ and $1330-\mathrm{keV}$ photon energies are shown in Table 1, and those for all oxide samples are plotted in Figure 2. It can be observed from the figure and table that $\mu$ decreases with increasing photon energy. It is observed that he experimental values of $\mu$ agree with the theoretical values calculated using the XCOM program.

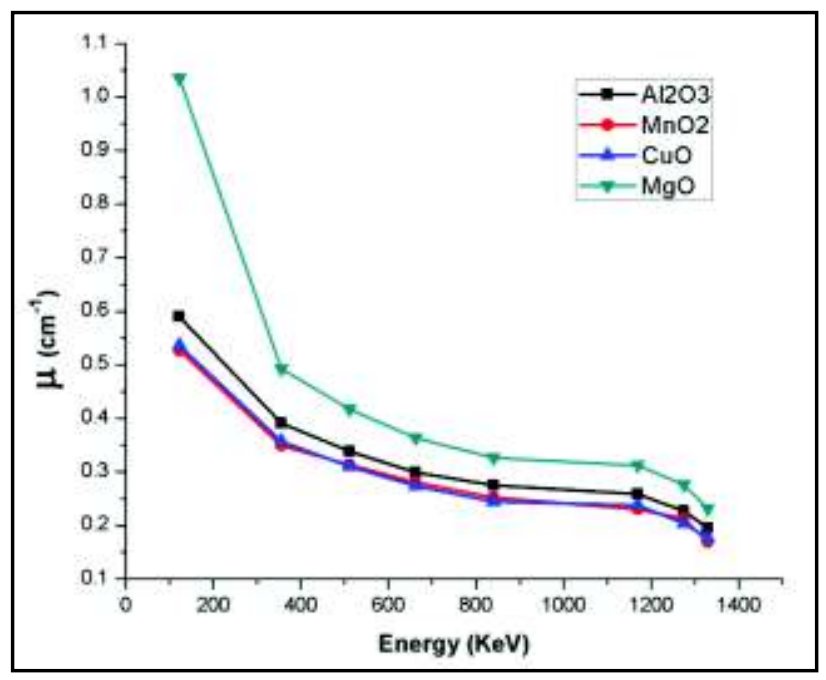

Figure 2: Typical Plot of $\mu$ versus Energy for Oxide

The total uncertainties in experimental values of $\mu$ depend on the uncertainties of $I_{0}$ (without attenuation), $I$ (after attenuation) measurements of mass thickness values, and counting statistics. The estimated total uncertainty in the measured experimental values of $\mu$ was found to be in the range of $3-4 \%$. The another important parameter The half-value layers, tenth-value layers and mean free paths of four oxide sample materials for different radiation energies levels from $122 \mathrm{KeV}$ to $1330 \mathrm{KeV}$ have been obtained and are presented in Table 2, 3 and 4 respectively and those for plotted in figure 3, 4 and 5. The mean free path, half value layer and tenth value layer of oxide increases with increases in energy of photon. It is found that the lower the values of half-value layers and tenth-value layers, the higher are the radiation shielding material in terms of the thickness requirements. 
DAHINDE AND PAWAR: ANALYSIS OF HALF VALUE LAYER (HVL), TENTH VALUE LAYER (TVL) AND MEAN...

Table 1: Linear Attenuation Coefficient $(\mu)$ of Oxides

\begin{tabular}{|c|c|c|c|c|c|c|c|c|}
\hline Energy & \multicolumn{2}{|c|}{$\mathbf{A l}_{\mathbf{2}} \mathbf{O}_{3}$} & \multicolumn{2}{c|}{ MnO$_{2}$} & \multicolumn{2}{c|}{ CuO } & \multicolumn{2}{c|}{ MgO } \\
\hline Kev & Expt. & Theo. & Expt. & Theo. & Expt. & Theo. & Exp. & Theo. \\
\hline 122 & 0.5821 & 0.5901 & 0.522 & 0.5364 & 1.7163 & 1.7415 & 1.0202 & 1.0353 \\
\hline 356 & 0.3787 & 0.3907 & 0.3492 & 0.3564 & 0.6499 & 0.631 & 0.4774 & 0.4925 \\
\hline 511 & 0.3276 & 0.3388 & 0.3132 & 0.3096 & 0.5363 & 0.5232 & 0.4272 & 0.4171 \\
\hline 662 & 0.291 & 0.299 & 0.2808 & 0.2736 & 0.448 & 0.4606 & 0.3769 & 0.3638 \\
\hline 840 & 0.2751 & 0.2751 & 0.252 & 0.2448 & 0.4227 & 0.4101 & 0.3166 & 0.3266 \\
\hline 1170 & 0.2511 & 0.2591 & 0.2304 & 0.2376 & 0.3849 & 0.3975 & 0.3015 & 0.3116 \\
\hline 1275 & 0.2352 & 0.2272 & 0.2166 & 0.2038 & 0.347 & 0.3344 & 0.2613 & 0.2764 \\
\hline 1330 & 0.2083 & 0.1953 & 0.1692 & 0.1764 & 0.2587 & 0.2776 & 0.2412 & 0.2311 \\
\hline
\end{tabular}

Table 2: Half Value Layer (HVL) Thickness $X_{h}(\mathrm{~cm})$ for Oxides

\begin{tabular}{|c|c|c|c|c|c|c|c|c|}
\hline Energy & \multicolumn{2}{|c|}{$\mathbf{A l}_{\mathbf{2}} \mathbf{O}_{\mathbf{3}}$} & \multicolumn{2}{c|}{$\mathbf{M n O}_{2}$} & \multicolumn{2}{c|}{$\mathbf{C u O}$} & \multicolumn{2}{c|}{ MgO } \\
\hline Kev & Expt. & Theo. & Expt. & Theo. & Expt. & Theo. & Exp. & Theo. \\
\hline 122 & 1.1905 & 1.1744 & 1.3276 & 1.2919 & 0.4038 & 0.3979 & 0.6793 & 0.6694 \\
\hline 356 & 1.8299 & 1.7737 & 1.9845 & 1.9444 & 1.0661 & 1.0983 & 1.4516 & 1.4071 \\
\hline 511 & 2.1154 & 2.0455 & 2.2126 & 2.2384 & 1.2922 & 1.3245 & 1.6222 & 1.6615 \\
\hline 662 & 2.3814 & 2.3177 & 2.4679 & 2.5329 & 1.5469 & 1.5046 & 1.8387 & 1.9049 \\
\hline 840 & 2.5191 & 2.5191 & 2.7511 & 2.8309 & 1.6395 & 1.6898 & 2.1889 & 2.1219 \\
\hline 1170 & 2.7599 & 2.6746 & 3.0078 & 2.9167 & 1.8005 & 1.7434 & 2.2985 & 2.2241 \\
\hline 1275 & 2.9464 & 3.0502 & 3.2994 & 3.4004 & 1.9971 & 2.0724 & 2.5521 & 2.5072 \\
\hline 1330 & 2.4989 & 3.5484 & 4.0057 & 3.9286 & 2.5788 & 2.4964 & 2.9231 & 2.9987 \\
\hline
\end{tabular}

Table 3: Tenth Value Layer (TVL) Thickness $X_{t}(\mathrm{~cm})$ for Oxides

\begin{tabular}{|c|c|c|c|c|c|c|c|c|}
\hline Energy & \multicolumn{2}{|c|}{$\mathbf{A l}_{\mathbf{2}} \mathbf{O}_{3}$} & \multicolumn{2}{c|}{$\mathbf{M n O}_{2}$} & \multicolumn{2}{c|}{ CuO } & \multicolumn{2}{c|}{ MgO } \\
\hline Kev & Expt. & Theo. & Expt. & Theo. & Expt. & Theo. & Exp. & Theo. \\
\hline 122 & 3.9557 & 3.9021 & 4.4111 & 4.2927 & 1.3416 & 1.3222 & 2.2571 & 2.2241 \\
\hline 356 & 6.0803 & 5.8935 & 6.5939 & 6.4607 & 3.5431 & 3.6491 & 4.8232 & 4.6753 \\
\hline 511 & 7.0287 & 6.7963 & 7.3519 & 7.4373 & 4.2935 & 4.4011 & 5.39 & 5.5205 \\
\hline 662 & 7.9127 & 7.701 & 8.2001 & 8.4159 & 5.1397 & 4.9991 & 6.1093 & 6.3293 \\
\hline 840 & 8.3701 & 8.3701 & 9.1373 & 9.4061 & 5.4474 & 5.6147 & 7.2729 & 7.0502 \\
\hline 1170 & 9.1701 & 8.8869 & 9.9941 & 9.6911 & 5.9823 & 5.7927 & 7.6371 & 7.3896 \\
\hline 1275 & 9.7901 & 10.135 & 10.9311 & 11.298 & 6.6357 & 6.8858 & 8.5581 & 8.3306 \\
\hline 1330 & 11.454 & 11.791 & 13.2019 & 13.053 & 8.4007 & 8.2947 & 9.7464 & 9.9637 \\
\hline
\end{tabular}

Table 4: Mean Free Path (Mfp) Thickness $X_{m}(\mathbf{c m})$ for Oxides

\begin{tabular}{|c|c|c|c|c|c|c|c|c|}
\hline Energy & \multicolumn{2}{|c|}{$\mathbf{A l}_{2} \mathbf{O}_{3}$} & \multicolumn{2}{c|}{$\mathbf{M n O}_{2}$} & \multicolumn{2}{c|}{ CuO } & \multicolumn{2}{c|}{ MgO } \\
\hline Kev & Expt. & Theo. & Expt. & Theo. & Expt. & Theo. & Exp. & Theo. \\
\hline 122 & 1.7179 & 1.6946 & 1.9157 & 1.8643 & 0.9802 & 0.9659 & 0.5826 & 0.5742 \\
\hline 356 & 2.6406 & 2.5595 & 2.8637 & 2.8058 & 2.0947 & 2.0305 & 1.5387 & 1.5848 \\
\hline 511 & 3.0525 & 2.9516 & 3.1928 & 3.2301 & 2.3408 & 2.3975 & 1.8646 & 1.9113 \\
\hline 662 & 3.4364 & 3.3445 & 3.5613 & 3.6551 & 2.6532 & 2.7488 & 2.2321 & 2.1711 \\
\hline 840 & 3.6351 & 3.6351 & 3.9683 & 4.0851 & 3.1586 & 3.0618 & 2.3657 & 2.4384 \\
\hline 1170 & 3.9825 & 3.8595 & 4.3403 & 4.2088 & 3.3167 & 3.2092 & 2.5981 & 2.5157 \\
\hline 1275 & 4.2517 & 4.4014 & 4.8268 & 4.9068 & 3.7171 & 3.6179 & 2.8818 & 2.9904 \\
\hline 1330 & 4.9988 & 5.1203 & 5.7102 & 5.6689 & 4.2459 & 4.3271 & 3.7054 & 3.6023 \\
\hline
\end{tabular}




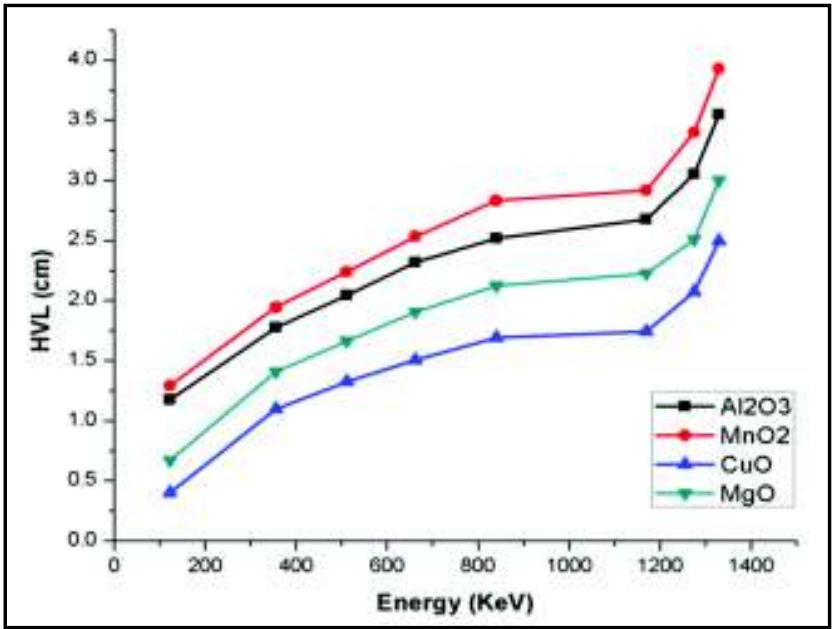

Figure 3: Typical Plot of HVL Thickness versus Energy

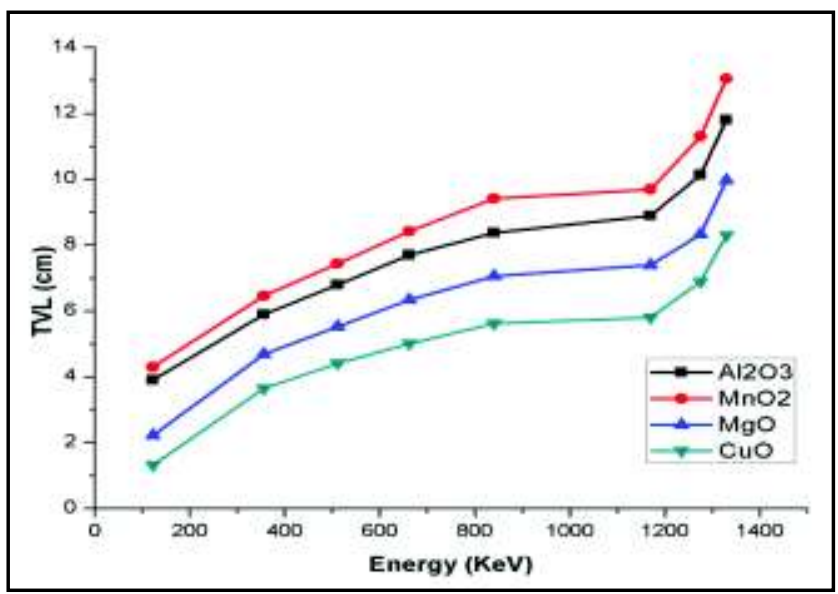

Figure 4: Typical Plot of TVL Thickness versus Energy for Oxide

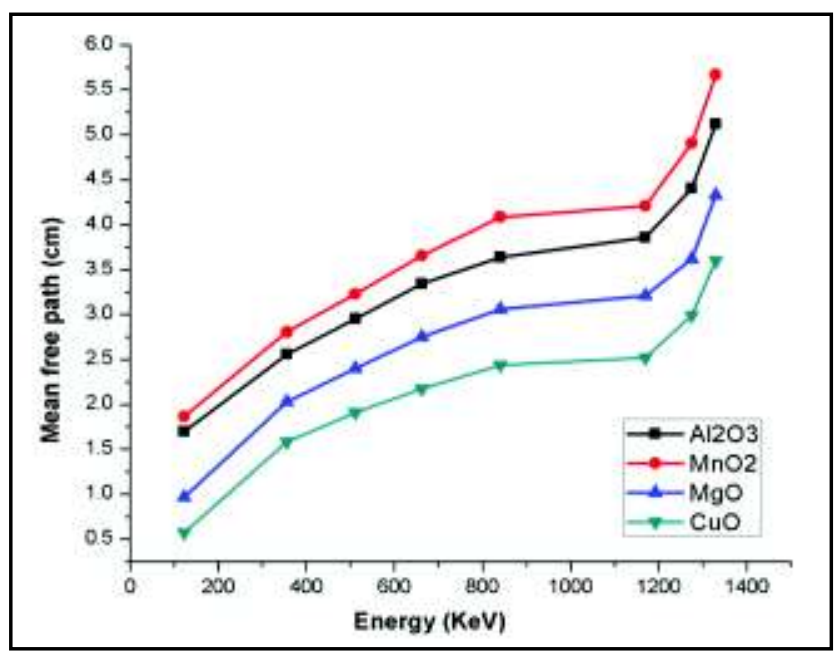

Figure 5: Typical Plot of Mean Free Path versus Energy of Oxide

\section{CONCLUSION}

The present experimental study was carried out to obtain information on linear attenuation coefficient, $\mu$ and related parameters such as half value layer (HVL), tenth value layer (TVL) and mean free path (mfp) for the selected four oxide samples. It has been found that $\mu$ is an extremely useful and sensitive physical quantity for the determination of these parameters for the chosen oxide samples. such as aluminum oxide $\left(\mathrm{Al}_{2} \mathrm{O}_{3}\right)$, manganese oxide $\left(\mathrm{MnO}_{2}\right)$, copper oxide $(\mathrm{CuO})$, magnesium oxide $(\mathrm{MgO})$, are determined in the chosen energy range $(122-1330 \mathrm{keV})$ which is emitted by the radioisotopes ${ }^{60} \mathrm{Co},{ }^{57} \mathrm{C}_{\mathrm{o}},{ }^{133} \mathrm{Ba},{ }^{54} \mathrm{Mn},{ }^{22} \mathrm{Na}$, and ${ }^{137} \mathrm{Cs}$. For the interaction of photons with matter the values of that $\mu$ depend on the physical and chemical environments of the samples. It is found that from table and figure these values decrease with increasing photon energies. From the study it is clear that it depends totally on the number and nature of atoms. In the present work, it has been observed that the data on linear attenuation coefficient $(\mu)$ and other parameters are HVL, TVL and $\mathrm{mfp}$ are very useful in industrial, biological, technological, shielding and other applications, solar cell and recently in sensors field. The measured data were compared against Win-XCOM- based data the agreement within 3-4\%.

\section{ACKNOWLEDGMENT}

The authors thank to Prof. G. K. Bichile for his fruitful discussion on this study and Dept. of physics, Dr. Babasaheb Ambedkar Marathvada University, Aurangabad.

\section{REFERENCES}

Akkaş A., 2015. Determination of the Tenth and Half Value Layer Thickness of Concretes with Different Densities, 5th International Science Congress \& Exhibition APMAS2015, Lykia, Oludeniz, pp.1619.

Akkurt I., Akyıldırım H., Mavi B., Kilincarslan S. and Basyigit C., 2010. Ann. Nucl. Energy, 37: 910.

Berger M.J. and Hubbell J.H., 1987/1999. "XCOM: Photon Cross Section Database," Wb Version 1.2, available at http:// Physics.nist.gov/XCOM. National Institute of standards and Technology, Gaithersburg, MD 20899, USA (1999). Originally published as NBSIR 87-3597 "XCOM: Photon Cross Sections on a Personal Computer". 
DAHINDE AND PAWAR: ANALYSIS OF HALF VALUE LAYER (HVL), TENTH VALUE LAYER (TVL) AND MEAN...

Bashter I.I., 1997. Calculation of radiation coefficients foe shielding concrete. Ann. Nuclear Energy, 24(17):1389-1401.

Creagh D.C., 1987. The resolution of discrepancies in tables of photon attenuation coefficients Nucl Instrum Methods A255, 1-16.

Gundogdua O., Tarimb U.A. and Gurlerb O., 2016. Monte Carlo Calculations for Photon Attenuation Studies on Different Solid Phantom Materials, (ICCESEN).
Hubbell J.H., 1999. Review of photon interaction cross section data in the medical and biological context Phys. Med. Biol., 44 R1-22.

Hubbell J.H. and Seltzer S.M., 1995. NIST (IR) Report No. 5632.

Jackson D.F. and Hawkes D.J., 1981. X-ray attenuation coefficients of elements and mixtures Phys. Rep., 70: $169-233$.

Singh N., Singh K.J., Singh K. and Singh H., 2006. Radiat. Meas., 41: 84. 